\title{
Block Split Subdivision Domination in Graphs
}

\author{
M. H. Muddebihal ${ }^{\text {* }}$, P. Shekanna' ${ }^{2}$ S. Ahmed ${ }^{3}$ \\ ${ }^{1,3}$ Professor,Department of Mathematics, Gulbarga University, Gulbarga-585106,Karnataka, \\ India \\ ${ }^{2}$ Research Scholar, Department of Mathematics, Gulbarga University, Gulbarga-585106, \\ Karnataka, India
}

Received 3 April 2015, accepted in final revised form 28 July 2015

\begin{abstract}
A dominating set $D \subseteq V[B S(G)]$ is a split dominating set in [BS(G)], if the induced subgraph $\langle V[B S(G)]-D\rangle$ is disconnected in $[B S(G)]$. The split domination number of $[B S(G)]$ is denoted by $\gamma_{s b \vec{s}}(G)$, is the minimum cardinality of a split dominating set in [BS(G)]. In this paper, some results on $\gamma_{g b s}(G)$ were obtained interms of vertices, blocks and other different parameters of $\mathrm{G}$ but not the members of $[B S(G)]$. Further we developed its relationship with other different domination parameters of $G$.
\end{abstract}

Keywords: Domination; Subdivision graph; Block subdivision graph; Split domination number.

(C) 2015 JSR Publications. ISSN: 2070-0237 (Print); 2070-0245 (Online). All rights reserved.

doi: http://dx.doi.org/10.3329/jsr.v7i3.22857 J. Sci. Res. 7 (3), 43-51 (2015)

\section{Introduction}

All graphs, subdivision graphs and block subdivision graphs considered here are simple, finite, nontrivial, undirected and connected, as usual $p, q, n$ denotes the number of vertices, edges and blocks of a graph $G$ respectively. In this paper, the study of domination in graphs started by C. Berge [1] and O. Ore [2]. The graph theoretical terminology undefined or notations can be found in G. Chartrand and P. Zhang [3] and F. Harary [4]. The domination in graphs is discussed by T. W. Haynes [5].

As usual, the minimum degree and maximum degree of a graph $G$ are denoted by $\delta(G)$ and $\Delta(G)$ respectively. A vertex $v$ is called a cut vertex if removing it from $G$

\footnotetext{
* Corresponding author: mhmuddebihal@yahoo.com
} 
increases the number of components of $G$. For any real number $x_{v}\lfloor x\rfloor$ denotes the greatest integer not greater than $x,\lceil x\rceil$ denotes the smallest integer not less than $x$.

The complement $\bar{G}$ of a graph $G$ has $V$ as its vertex set, but two vertices are adjacent in $\bar{G}$, if they are not adjacent in $G$. The vertex independence number $\beta_{0}(G)$ is the maximum cardinality of an independence set of vertices. An edge cover of $G$ is the set of edges that covers all the vertices. The edge covering number $a_{1}(G)$ is minimum cardinality of an edge cover. The edge independence number $\beta_{1}(G)$ is the minimum cardinality of an independent set of edges. A set of vertices $D \subseteq V(G)$ is a dominating set, if every vertex in $V-D$ is adjacent to some vertex in $D$. The dominance number $\gamma(G)$ of $G$ is the minimum cardinality of a dominating set in $G$ [6]. A dominating set $D$ of $G$ is a cototal dominating set, if the induced sub graph $\langle V-D\rangle$ has no isolated vertices. The cototal domination number $\gamma_{\text {cot }}(G)$ of $G$ is the minimum cardinality of a cototal dominating set [6]. A dominating set $D$ of a graph $G$ is a split dominating set, if the induced subgraph $\langle V-D\rangle$ is disconnected. The split domination number $\gamma_{s}(G)$ of a graph $G$ is the minimum cardinality of a split dominating set. This concept was introduced by Kulli [6,7]. A dominating set $D$ is a connected dominating set, if the induced sub graph $\langle D\rangle$ is connected. The connected domination number $\gamma_{c}(G)$ of a connected graph $G$ is the minimum cardinality of a connected dominating set [8]. The dominating set $D$ is a total dominating set, if the induced subgraph $\langle D\rangle$ has no isolated vertices. The total domination number $\gamma_{\mathrm{t}}(G)$ of a graph $G$ is the minimum cardinality of a total dominating set [9].

The following figures illustrate the formation of $[B S(G)]$ of a graph $G$.
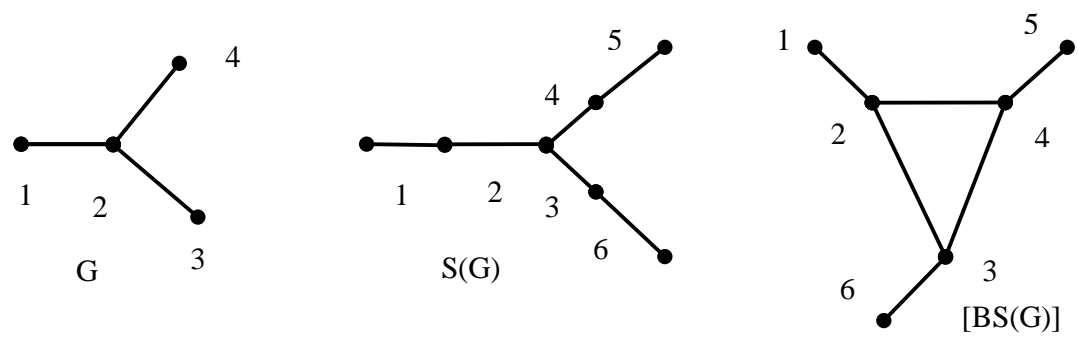

Blocks of $\mathrm{G}=\mathrm{n}=3$

The domination of block split subdivision domination in graph is denoted by $\gamma_{s b s}(G)$. In this paper some results on $\gamma_{s b s}(G)$ were obtained interms of vertices, blocks and other different parameters of $G$.

One application of block split subdivision domination is that of prisoners and guards. For security and their over all development of prisoners, each prisoner must be seen by some guards, the concept is that of domination. However in order to develop good attitudes of prisoners, we may require that of subdivision graph, also require each prisoner is taken care by the higher official for overall development, the concept is that of block split subdivision domination.

We need the following theorems for our further results. 


\section{Main Results}

Here, we will consider several domination parameters and their relation to the block split subdivision domination number. The following results appeared in [6].

Theorem 1: A split dominating set $D$ of $G$ is minimal of each vertex. $v \in D_{x}$ One of the following conditions holds.

i) There exists a vertex $u \in V-D$, such that $N(u) \cap D=\{v\}$.

ii) $v$ is an isolated vertex in $\langle D\rangle$.

iii) $\langle(V-D) \cup\{v\}\rangle$ is connected.

Theorem 2: For any graph $\gamma_{s}(G) \leq \frac{p \Delta(G)}{1+\unlhd(G)}$.

\section{Upper bound for $\gamma_{s b s}(G)$}

In this section we compare the parameter $\gamma_{s b s}(G)$ with the blocks, domination and end blocks of $G$.

Theorem 3: For any connected graph $G$ with $n$-blocks and $[B S(G)] \neq K_{p}$, then $\gamma_{s b s}(G) \leq n$.

Proof: Suppose $[B S(G)]$ is a complete graph. Then by definition, split domination does not exists. Hence $[B S(G)] \neq K_{p}$.

Let $S=\left\{B_{1 \mathrm{a}} B_{2}, B_{2}, \ldots \ldots \ldots B_{n}\right\}$ be the number of blocks in $G$ and $S_{1}=\left\{B_{1}{ }^{1} B_{2}{ }^{1}, B_{a}{ }^{1}, \ldots \ldots \ldots B_{m}{ }^{1}\right\}$ be the blocks in $S(G)$. We consider the following cases.

Case 1: Suppose $\forall B_{i} \in S$ there exists atleast one $B_{i}, 1 \leq i \leq n$ as an edge. Let $V[B S(G)]=\left\{b_{1}{ }^{1} b_{2}{ }^{1}, b_{a}{ }^{1}, \ldots \ldots m, b_{m}{ }^{1}\right\}$ be a set of vertices corresponding to the blocks of $S(G)$. In $S_{1}, \forall B_{i} \in S$ generates $B_{i}{ }^{1}$ and $B_{j}{ }^{1}$ as blocks in $S(G)$ such that $\forall B_{i}{ }^{1}{ }_{x} B_{j}{ }^{1} \in S$. Clearly $b_{i}{ }^{1}, b_{j}{ }^{1} \in V[B S(G)]$. Consider $V_{1} \subset V[B S(G)]$ and $V_{2}=V[B S(G)]-V_{1}$. If every vertex of $V_{2}$ is adjacent to at least one vertex in $V_{1}$ and if there exist more than one component in $V_{2}$, then $V_{1}$ is a split dominating set. Further if $V_{2} \cup\left\{b_{k}\right\}$ where $b_{k} \in V_{1}$ gives one component in $[B S(G)]-V_{1}$. Then $V_{1}$ is a minimal split dominating set of [BS(G)]. Hence $\left|V_{1}\right|=\gamma_{s b s}(G)-$ set which gives $\gamma_{s b s}(G) \leq n$.

Case 2: Suppose $\forall B_{i} \in S$ such that each $B_{\mathrm{i}}$ is an edge. Then $S_{1}=S+\{q\}$ where $\{q\}$ is the number of edges in $S(G)$. As in case 1, which gives $\gamma_{s b s}(G) \leq n$.

Next we discuss in case 3, about the blocks of $G$ such that each block contains at least three vertices. 
Case 3: Suppose $\forall B_{i} \in S$ such that each $B_{i}$ is not an edge. Let $V[B S(G)]=\left\{b_{1}{ }^{1}, b_{2}{ }^{1}, b_{a}{ }^{1} \ldots \ldots \ldots, b_{m}{ }^{1}\right\}$ be the set of vertices corresponding to the blocks of $S(G)$. Consider $V_{1} \subset V[B S(G)]$ and $V_{2}=V[B S(G)]-V_{1}$. If every vertex of $V_{2}$ adjacent to at least one vertex in $V_{1}$ and $V_{2}$ has more than one component, then $\left|V_{1}\right|$ gives the split domination number by the definition of block graph which is always less than the number of blocks in $G$. Hence $\left|V_{1}\right| \leq \mathrm{n}$ which gives $\gamma_{\text {sbs }}(G) \leq n$.

Theorem 4: For any graph $(p, q)$ with $N$-end blocks and $[B S(G)] \neq K_{p}$ then $\gamma_{s b s}(G) \leq \gamma(G)+N$.

Proof: In view of the definition, we consider only those graphs which are not complete in [BS(G)]. Let $V=\left\{v_{1}, v_{2}, v_{a}, \ldots \ldots \ldots, v_{n n}\right\}$ be the vertices in $G$ and $V_{1} \subset V_{x}$ every vertex of $V-V_{1}$ is adjacent to atleast one vertex of $V_{1}$, then $\left|V_{1}\right|=\gamma(G)$. To establish the upper bound for $\gamma_{s b s}(G)$ in terms of $\gamma(G)$ and the number of end blocks in $G$. We consider the following cases.

Case 1: Suppose each block of $G$ is an edge. Then in $S(G)$ the number the blocks is $2 q$. Let $S=\left\{B_{1}, B_{2}, B_{2}, \ldots \ldots \ldots B_{n}\right\}$ be the block in $G$. In $S(G)$, $\left\{B_{1}{ }^{1} B_{2}{ }^{1}, B_{a}{ }^{1}, \ldots \ldots \ldots B_{m}{ }^{1}\right\} \cup\left\{B_{1}, B_{2}, B_{a}, \ldots \ldots \ldots B_{n}\right\}$ are the blocks. Now $V[B S(G)]=\left\{b_{1}, b_{2}, b_{a}, \ldots \ldots \ldots b_{n}\right\} \cup\left\{b_{1}{ }^{1} b_{2}{ }^{1}, b_{a}{ }^{1}, \ldots \ldots \ldots, b_{m}{ }^{1}\right\}$ corresponds to the blocks of $S(G)$. Let $H_{1}=\left\{b_{1}, b_{2}, b_{a}, \ldots \ldots \ldots b_{i}\right\}, 1 \leq i \leq n_{n} H_{2}=\left\{b_{1}{ }^{1}, b_{2}{ }^{1}, b_{a}{ }^{1}, \ldots \ldots \ldots b_{j}\right\} 1 \leq j \leq m$ and $\left\{H_{1} \cup H_{2}\right\} \subseteq V[B S(G)]$ are the cut vertices. Suppose $H_{1}^{1} \subseteq H_{1}$ and $H_{2}^{1} \subseteq H_{2}$ and $D=V[B S(G)]-\left\{H_{1}^{1} \cup H_{2}^{1}\right\}$. Then $\forall b_{i} \in V[B S(G)]-\left\{H_{1}^{1} \cup H_{2}^{1}\right\}$ are adjacent at least one vertex of $D$ gives $\left(V[B S(G)]-\left\{H_{1}^{1} \cup H_{2}^{1}\right\}\right)$ a disconnected graph. Hence $|D|$ is a $\gamma_{s b s}(G)-$ set. In, $S(G)$ the number of blocks is more than that of $G$. On comparing the cardinality of $V_{1}(G)$ and the number of end blocks $N$, we have $|D| \leq\left|V_{1}\right|+|N|$ which gives $\gamma_{s b s}(G) \leq \gamma(G)+N$.

Case 2: Suppose at least one block of $G$ is not an edge. Then there exists a block with atleast three vertices, for this block $\left|V_{1}\right|$ is more than as in case 1. Again $\left|\left\langle V[B S(G)]-\left\{H_{1}^{1} \cup H_{2}^{1}\right\}\right\rangle\right|$ remains constant.

Hence $|D| \leq\left|V_{1}\right|+|N|$ gives $\gamma_{s b s}(G) \leq \gamma(G)+N$.

Further we compared our concept to the domination and cut vertices of subdivision of G.

Theorem 5: For any connected $(p, q)$ graph $G$ and $[B S(G)] \neq K_{p}$, then $\gamma_{s b s}(G) \leq \gamma[S(G)]$.

Proof: For any connected graph $G$, by the definition of split domination $[B S(G)] \neq K_{p}$. We have the following cases. 
Case 1: Suppose each block of $G$ is an edge. Then $V[S(G)]=V(G)+\{q\}$. Let $V(G)=\left\{v_{1}, V_{2}, v_{n}, \ldots \ldots \ldots v_{n}\right\}$ and $[S(G)]-V(G)=\left\{v_{1}{ }^{1}, v_{2}{ }^{1}, v_{a}{ }^{1}, \ldots \ldots \ldots v_{m}{ }^{1}\right\}$. We consider $V_{1}(G)=\left\{v_{1}, v_{2}, v_{a}, \ldots \ldots \ldots v_{i}\right\}, 1 \leq i \leq n$ and $V_{2}(G)=\left\{v_{1}{ }^{1}, v_{2}{ }^{1}, v_{a}{ }^{1}, \ldots \ldots \ldots v_{j}{ }^{1}\right\}, 1 \leq j \leq m$. If there exists a set $D=V[S(G)]-\left\{V_{1}(G) \cup V_{2}(G)\right\}$ such that $\forall v_{k} \in D$ is adjacent to at least one vertex of $\left\{V_{1}(G) \cup V_{2}(G)\right\}$, then $\left\{V_{1}(G) \cup V_{2}(G)\right\}$ is $\gamma[S(G)]-$ set. Since each edge is a block in $G$, then $V[B S(G)]=E(G)+\{q\}$ and $V[B S(G)] \leq V[S(G)]$. Let $H=\left\{v_{11_{3}} v_{2}, v_{a^{\prime}}, \ldots \ldots \ldots v_{k}\right\}$ be the block vertices corresponding to the blocks of $S(G)$ such that $H \subset V[B S(G)]$. Suppose $l=V[B S(G)]-H$ and $\forall v_{j} \in H$ are adjacent to at least one vertex of $l$ and generates more than one component in $l$. Then $H$ is a $\gamma_{s b s}(G)-$ set. Hence $|H| \leq\left|V_{1}(G) \cup V_{2}(G)\right|$ gives $\gamma_{s b s}(G) \leq \gamma[S(G)]$.

Case 2: Suppose $G$ has at least one block which is not an edge. Then there exists a block $B$ with at least three edges. Clearly $V[S(G)]>V[B S(G)]$. In this view and as the argument in case 1 , we have $\gamma_{s b s}(G) \leq \gamma[S(G)]$.

Theorem 6: Let $G$ be a connected graph with $C$ number of cut vertices of $S(G)$ and $[B S(G)] \neq K_{p}$, then $\gamma_{s b s}(G) \leq\left\lceil\frac{C[S[G)]}{2}\right]$.

Proof: Suppose $[B S(G)] \neq K_{p}$. Then we considered the following cases.

Case 1: Suppose $G$ is acyclic graph. Let $s=\left\{B_{1_{a}} B_{2}, B_{a^{2}, \ldots \ldots \ldots} B_{n}\right\}$ be the blocks of $G$. In $S(G), S_{1}=\left\{B_{1,} B_{2}, B_{a}, \ldots \ldots \ldots B_{n}, B_{1}{ }^{1} B_{2}{ }^{1}, B_{a}{ }^{1} \ldots \ldots \ldots B_{m}^{1}\right\}$ are the blocks. Let $\left\{B_{1} B_{2}, B_{a}, \ldots \ldots \ldots B_{\mathrm{i}}, B_{1}{ }^{1} B_{2}{ }^{1}, B_{a}{ }^{1} \ldots \ldots \ldots B_{j}\right\}, 1 \leq i, j \leq n, m$ are the non end blocks of $S(G)$. Then in $B[S(G)]$, the set $H=\left\{b_{1}, b_{2}, b_{a}, \ldots \ldots \ldots b_{i}, b_{1}{ }^{1}, b_{2}{ }^{1}, b_{a}{ }^{1}, \ldots \ldots \ldots b_{j}{ }^{1}\right\}$ is the cut vertex set. Now there exists a set $H_{1} \subset H$ such that $H_{2}=V[B S(G)]-H_{1}$ and $\forall b_{i} \in H_{1}$ are adjacent to all the vertices of $V[B S(G)]-H_{1}$. Since $\forall b_{i} \in H$ is a cut vertex, then $\left\langle V[B S(G)]-H_{1}\right\rangle$ is

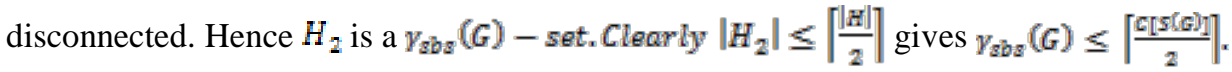

Case 2: Suppose $G$ is cyclic graph. Then it contains at least one block which is cyclic. In $S(G)$ the blocks remains same as in $G$ and $V[S(G)] \geq V(G)$. Let $M \subset V[B S(G)]$ is a cut vertex set, there exist a set $M_{1} \subset M$ such that $M_{2}=V[B S(G)]-M_{1} \forall b_{i} \in M_{1}$ is adjacent to all the vertices of $V[B S(G)]-M_{1 \times}$ Since $\forall b_{i} \in M$ is a cut vertex, then $\left\langle V[B S(G)]-M_{1}\right\rangle$ has more than one component. Hence $M_{2}$ is a $\gamma_{s b s}(G)-$ set. Clearly $\left|M_{2}\right| \leq\left\lceil\frac{|M|}{2}\right\rceil$ gives $\gamma_{s b s}(G) \leq\left\lceil\frac{C[S(G)]}{2}\right\rceil$.

Now we proceed to establish the upper bound interms of $\Delta(G)$, minimum, vertex and edge covering of $G$ and maximum independence number of $G$ for block split subdivision domination of $G$.

Theorem 7: For any graph $G,[B S(G)] \neq K_{p}$ then $\gamma_{s b s}(G) \leq\left\lfloor\frac{p \Delta(G)}{1+\unlhd(G)}\right\rfloor$. 
Proof: To establish the required results, we have the graphs $[B S(G)] \neq K_{p}$. Let $V=\left\{V_{1}, v_{2}, v_{a}, \ldots \ldots \ldots v_{n}\right\}$ be the vertices of $G$ and $S_{1}=\left\{B_{1}{ }^{1} B_{2}{ }^{1}, B_{a}{ }^{1}, \ldots \ldots \ldots B_{m}{ }^{1}\right\}$ be the blocks in $S(G)$. Let $V[B S(G)]=\left\{b_{1}{ }^{1}, b_{2}{ }^{1}, b_{a}{ }^{1}, \ldots \ldots m b b_{m}{ }^{1}\right\}$ be the vertices in [BS(G)] which corresponds to the blocks of $S_{1}$. Let $D$ be a dominating of $[B S(G)]_{\text {s suppose }} \forall v_{i} \in D$, there exists a vertex $u \in V[B S(G)]-D$ such that $N(u) \cap D=\left\{v_{i}\right\}$ and $V[B S(G)]-D$ has more than one component.

Now for a vertex $v \in V(G)$ with $\operatorname{deg}(v)=\Delta(G) . \quad\{D\}\{1+\Delta(G)\} \leq V(G) \cdot \Delta(G)$. Further $\{D\} \leq \frac{V(G) \Delta(G)}{[1+\Delta(G)]}$ and $|D| \leq\left|\frac{V(G) \Delta(G)}{1+\Delta(G)}\right|$ gives $\gamma_{g b s}(G) \leq\left|\frac{p \Delta(G)}{1+\Delta(G)}\right|$.

Theorem 8: For any graph $G_{v}[B S(G)] \neq K_{p}$ then $\gamma_{s b b s}(G) \leq \alpha_{1}(G)+\Delta(G)-1$ where $\alpha_{1}(G)$ is the minimum edge covering number of $G$.

Proof: By the definition of split domination, $[B S(G)] \neq K_{p}$. Let $S=\left\{B_{1_{a}} B_{2}, B_{a}, \ldots \ldots \ldots B_{n}\right\}$ the blocks of $G$ and $S_{1}=\left\{B_{1}{ }^{1} B_{2}{ }^{1}, B_{a}{ }^{1}, \ldots \ldots \ldots B_{m}{ }^{1}\right\}$ be the blocks in $S(G)$. Let $V=\left\{b_{1}{ }^{1} b_{2}{ }^{1}, b_{3}{ }^{1}, \ldots \ldots b_{m}{ }^{1}\right\}$ be the set of vertices in [BS(G)] which corresponds to the blocks of $S_{1}$. Let $B=\left\{e_{1_{a}} e_{2}, \theta_{x} \ldots \ldots \ldots e_{n}\right\} \subset E(G)$ and $V=\left\{v_{1} v_{2}, v_{a}, \ldots \ldots \ldots v_{n}\right\}$ be the set of vertices which are incident with the edges of $B$ and if $|V|=P_{x}$ then $B$ itself is an edge covering number. Otherwise, consider the minimum number of edges $\left\{e_{m}\right\} \subset E(G)-B$, such that $B_{1}=B \cup\left\{e_{m}\right\}$ forms a minimal edge covering set of $G$. Suppose $V_{1}[B S(G)] \subset V[B S(G)], \forall v_{i} \in V_{1}[B S(G)]$ which are cut vertices in $[B S(G)]$. Again $V_{2}[B S(G)] \subset V_{1}[B S(G)] \forall v_{j} \in V_{2}[B S(G)]$. Let $V_{a}[B S(G)]$ be the end vertices in [BS(G)]. Then $N\left(V_{2}\right) \cap N\left(V_{a}\right) \neq \emptyset_{x} \quad V[B S(G)]-\left\{V_{2}[B S(G)] \cup V_{a}[B S(G)]\right\}=H_{x}$ every vertex in $\left(V_{2} \cup V_{a}\right)$ is adjacent to atleast one vertex in $H$ and $\left\langle V_{2} \cup V_{a}\right\rangle$ has more than one component. Then $(H)$ is a split dominating set, $|H|=\gamma_{s b s}(G)$. Clearly $|H| \leq\left|B_{1}\right|+\Delta(G)-1$ which gives $\gamma_{s b s}(G) \leq \alpha_{1}(G)+\Delta(G)-1$.

Theorem 9: Let $G$ be a graph with $[B S(G)] \neq K_{p}$, then $\gamma_{s b s}(G)+\gamma_{c}(G) \leq \alpha_{0}(G)+\beta_{0}(G)+\gamma(G)+1$. Equality holds if $G \cong P_{9}$.

Proof: To establish the required result we have the graph $[B S(G)] \neq K_{p}$. Let $V_{1}=\left\{v_{1,} v_{2}, v_{2}, \ldots \ldots \ldots v_{n}\right\} \subseteq V(G)$ be the set of vertices with $\operatorname{deg}\left(v_{i}\right) \geq 2, \forall v_{i} \in V_{1}, 1 \leq i \leq n$, which are at distance at most two, covers all the edges in $G$. Clearly $\left|V_{1}\right|=\alpha_{0}(G)$. Further, if for any vertex $x \in V_{1}, N(x) \in V(G)-V_{1}$, then $V_{1}$ itself is an independent vertex set, otherwise $V_{1}^{1} \cup V_{2}$, where $V_{1}^{1} \subseteq V_{1}$ and $V_{2} \subseteq V(G)-V_{1}$ forms a maximum independent set of $G$ with $\left|V_{1}^{1} \cup V_{2}\right|=\beta_{0}(G)$. Let $S=H \cup H_{1}$, where $H \subseteq V_{1}$ and $H_{1} \subseteq V(G)-V_{1}$ be the minimal set of vertices which covers all the vertices in $G$. Clearly $S$ forms a minimal $\gamma$-set of $G$. Suppose the subgraph $\langle s\rangle$ has more than one component, then attach the minimum number of vertices $\left\{W_{i}\right\} \in V(G)-S_{x}$ where $\operatorname{deg}\left(W_{i}\right) \geq 2$ which are between the vertices of $S$ such that $S_{1}=S \cup\left\{W_{1}\right\}$ forms exactly one component in the sub graph $\left\langle S_{1}\right\rangle$,clearly $S_{1}$ forms a minimal $\gamma_{c}-$ set of $G$. Let $M=\left\{v_{1_{1}} v_{2}, v_{a}, \ldots \ldots \ldots v_{n}\right\}$ be the set of vertices in $[B S(G)]$. If $l=\left\{v_{1,} v_{2}, v_{a}, \ldots \ldots \ldots v_{m}\right\}$ where $1 \leq m \leq n_{x}$ such that $l \subset M$ and 
suppose that there exists a set $J_{1} \subset J$, then $\left\{v_{i}\right\} \in J_{1}$ which gives $M-J_{1}$ is a disconnected graph. Suppose $l_{1} \cup J$ has the minimum number of vertices, such that $\left.N\left(g_{1} \cup j\right)=V[B S(G)]-O_{1} \cup J\right)$ gives a minimal split dominating in $[B S(G)]$. Hence $\left|J_{1} \cup j\right|=\gamma_{s b s}(G)$. Clearly $\left|j_{1} \cup j\right|+\left|S_{1}\right| \leq$ $\left|V_{1}\right|+\left|V_{1}^{1} \cup V_{2}\right|+|S|+1$ which gives $\gamma_{s b s}(G)+\gamma_{c}(G) \leq \alpha_{0}(G)+\beta_{0}(G)+\gamma(G)+1$.

For equality, If $\mathrm{G}=P_{9}$, then $\gamma\left(P_{9}\right)=3, \alpha_{0}\left(P_{9}\right)=4, \beta_{0}\left(P_{9}\right)=5, \gamma_{0}\left(P_{9}\right)=7$. But we have $\gamma_{s b s}\left(P_{9}\right)=6$, which satisfies the equality of the statement of theorem.

Theorem 10: For any connected graph $G$ with $n$-blocks and $[B S(\bar{G})] \neq K_{p}$, then $\gamma_{s b s}(\bar{G}) \leq n$.

Proof: using Theorem 1, the above result follows.

\section{Lower bound for $\gamma_{s b s}(G)$}

In this section we will show that the sharp lower bound to the cototal domination, total domination and maximum independence (vertex/edge) number of $G$.

Theorem 11: For any connected graph $G$ and $[B S(G)] \neq K_{p}$, then $\gamma_{s b s}(G)+\Delta(G) \geq \gamma_{c o t}(G)+2$.

Proof: We consider only $[B S(G)] \neq K_{p}$. For $\gamma_{\text {cot }}(G)$, we consider the following cases.

Case 1: Suppose $G$ has end vertices, Let $H=\left\{v_{1}, v_{2}, v_{a}, \ldots \ldots \ldots v_{g}\right\}$ be the set of vertices in $G$ and $H_{1}=\left\{v_{1}, v_{2}, v_{a}, \ldots \ldots \ldots v_{n}\right\}$ be the set of all end vertices and $H_{2}=\left\{v_{1}, v_{2}, v_{1}, \ldots \ldots \ldots, v_{k}\right\}$ be the set of all vertices at a distance three for each of $v_{n} \in H_{1}$. Further consider $H_{2}^{1} \subseteq H_{2}$ such that $\forall v_{i j}, v_{j} \in H_{2}^{1}, N\left(v_{i}\right) \cap N\left(v_{j}\right)=\emptyset$. Now $\left\langle V(G)-\left\{H_{1} \cup H_{2}^{1}\right\}\right\rangle$ has no isolates and every vertex of $\left(V(G)-\left\{H_{1} \cup H_{2}^{1}\right\}\right)$ is adjacent to at least one vertex of $\left\{H_{1} \cup H_{2}^{1}\right\}$. Hence $\left\{H_{1} \cup H_{2}^{1}\right\}$ is a $\gamma_{\text {cot }}(G)-$ set. Let $\left\{v_{i}\right\} \in V[B S(G)]$ is a set of cut vertices in $[B S(G)], V_{1} \subseteq V[B S(G)]$ then $V_{1}$ is a split dominating set. Otherwise $u_{i} \subset V[B S(G)]$ which are end vertices in $[B S(G)]$, then $V_{1} \cup\left\{u_{i}\right\}$ is a $\gamma-\operatorname{set}, \forall v_{i}, v_{j} \in V_{1}$ which are at a distance of three, $N\left(v_{i}\right) \cap N\left(v_{j}\right)=\emptyset$. Now $\left\langle V[B S(G)]-\left(V_{1} \cup\left\{u_{i}\right\}\right)\right\rangle$ is disconnected. Every vertex is adjacent to at least one vertex of $V_{1} \cup\left\{u_{i}\right\}$. Then $\left|V_{1} \cup\left\{u_{i}\right\}\right|=\gamma_{s b s}(G)$. Clearly $\| V_{1} \cup\left\{u_{i}\right\}|+\Delta(G) \geq| H_{1} \cup H_{2}^{1} \mid+2$ gives $\gamma_{s b s}(G)+\Delta(G) \geq \gamma_{c o t}(G)+2$.

Case 2: Suppose $G$ has no end vertices and each block is not an edge. Let $H \subset V(G)$ be a set of cut vertices in $G$ and $H_{1} \subset H, \forall v_{i} \in H_{1}$ which are at a distance two from each $v_{i}, v_{j} \in H_{1}$ Let $H_{2}=\left\{v_{s}\right\}$ be the set of non cutvertices in $G$. Again $H_{2}^{1} \subseteq H_{2}, \forall v_{t} \in H_{2}$ such that $N\left(v_{i}\right) \cap N\left(v_{t}\right)=\emptyset$. Now $\left\langle V(G)-\left\{H_{1} \cup H_{2}^{1}\right\}\right\rangle$ has no isolates and every vertex of $\left\langle V(G)-\left\{H_{1} \cup H_{2}^{1}\right\}\right\rangle$ is adjacent to at least one vertex of $\left\{H_{1} \cup H_{2}^{1}\right\}$. Hence $\left\{H_{1} \cup H_{2}^{1}\right\}$ is a $\gamma_{c o t}(G)-\operatorname{set}$. Since each block of $G$ has no edge, then $V[S(G)]>V[B S(G)]$ as in case 1 remains same, $\quad\left|V_{1} \cup\left\{u_{i}\right\}\right|=\gamma_{s b s}(G)$ which gives $\gamma_{s b s}(G)+\Delta(G) \geq \gamma_{c o t}(G)+2$. 
Theorem 12: Suppose $G$ be a graph and $[B S(G)] \neq K_{p}$, then $\gamma_{s b s}(G)+\Delta(G) \geq \gamma_{t}(G)$.

Proof: For split domination, we considered the graphs with the property such that $[B S(G)] \neq K_{p}$. we have the following cases.

Case 1: Suppose each block of $G$ is an edge. Then $P=q+1$. Let $V$ be the set of vertices in $G$ and $\quad V_{1} \subset V$ which are non end vertices in $G$. Again consider a subset $V_{2} \subset V_{1}$. If $V_{1}-V_{2}=D$ has no isolated vertices and $v_{i} \in V(G)-\left\{V_{1}-V_{2}\right\}$ has atleast one vertex adjacent to a vertex of $D$. Then $D$ is a total dominating set which gives $|D|=\gamma_{t}(G)$. Let $H=\left\{v_{1,} v_{2}, v_{2}, \ldots \ldots v_{n}\right\}$ be the cut vertices in $[B S(G)]$ which are non end blocks in $S(G)$. Consider $H_{1} \subset H, H-H_{1}=D_{1}, v_{i} \in H$. Then $V[B S(G)]-\left\{H-H_{1}\right\}$ is adjacent to at least one vertex in $V[B S(G)]$ and gives $\left\langle V[B S(G)]-\left\{H-H_{1}\right\}\right)$ a disconnected graph. Hence $\left|D_{1}\right|$ is $\gamma_{s b s}(G)-$ set. Clearly $\left|D_{1}\right|+\Delta(G) \geq|D|$ which gives $\gamma_{s b s}(G)+\Delta(G) \geq \gamma_{t}(G)$.

Case 2: Suppose each block of $G$ is not an edge. Then there exists at least one block which contains at least three vertices in $G$. Let $D_{2} \subset V(G)$ such that $\forall v_{i} \in V(G)-D_{2}$ is adjacent to at least one vertex of $D_{2}$ and $\forall v_{i} \in D_{2}$, $\operatorname{deg}\left(v_{i}\right) \geq 1$ which gives a total dominating set. Hence $\left|D_{2}\right|=\gamma_{t}(G)$. In $S(G)$, since each block contains atleast three vertices of $G$ the cardinality of blocks remains same as in $G$. As in case 1, again $\left\langle V[B S(G)]-\left\{H-H_{1}\right\}\right\rangle$ is disconnected. Hence $\left|D_{1}\right|+\Delta(G) \geq\left|D_{2}\right|$ which gives $\gamma_{s b s}(G)+\Delta(G) \geq \gamma_{t}(G)$.

Theorem 13: For any graph $G,[B S(G)] \neq K_{p}$ then $\gamma_{s b s}(G)+\gamma(G) \geq \beta_{0}(G)-1$ where $\beta_{0}(G)$ is a maximum independence number of $G$.

Proof: By the definition of split domination, $[B S(G)] \neq K_{p}$. Let $S=\left\{B_{1_{a}} B_{2}, B_{3}, \ldots \ldots \ldots B_{n}\right\}$ be the blocks of $G$ and $S_{1}=\left\{B_{1}{ }^{1} B_{2}{ }^{1}, B_{a}{ }^{1}, \ldots \ldots B_{n}{ }^{1}\right\}$ be the blocks in $5(G)$. Consider $\left\{b_{1}{ }^{1}, b_{2}{ }^{1}, b_{a}{ }^{1} \ldots \ldots \ldots b_{n}{ }^{1}\right\}$ be the set of vertices in [BS(G)] which corresponds to the blocks of $s_{1}$. We have the following cases.

Case 1: Suppose $G$ is a tree. Let $V_{1}=\left\{v_{1_{a}} v_{2}, v_{1}, \ldots \ldots \ldots v_{n}\right\}$ be the set of vertices in $G$ and $V_{2} \subset V_{1} \forall v_{i} \in V_{2}$ such that, $\exists e_{i} \in E(G)$ is joining two vertices of $V_{2}$. Then $\forall u, v \in\left\langle V(G)-V_{2}\right\rangle$, so $d(u, v) \geq 2$ and hence $\left|V(G)-V_{2}\right|=\beta_{0}(G)$. Suppose a set $H \subset V_{1}(G)$ be a minimal dominating set of $G$ such that, if every vertex in $V_{1}(G)-H$ is adjacent to atleast one vertex in $H$. Then $|H|=\gamma(G)$. Consider $H \subset V[B S(G)]$ which are cut vertices. Again $H_{1} \subset H \forall v_{i} \in H_{1}$ then there exists $\left\langle V[B S(G)]-H_{1}\right\rangle=\mathrm{J}$ a disconnected graph and every vertex in $I$ is adjacent to atleast one vertex of $H_{1}$. Then $\left|H_{1}\right|$ is a split domination number of [BS(G)]. Then $\left|H_{1}\right|=\gamma_{s b s}(G)$. Clearly $\left|H_{1}\right|+|H| \geq\left|V(G)-V_{2}\right|-1$ which gives $\gamma_{s b s}(G)+\gamma(G) \geq \beta_{0}(G)-1$.

Case 2: Suppose $G$ is not a tree. Then $G$ has atleast one block which is not an edge. Let $V_{1}(G)$ be the vertices in $G$ and $V_{2}^{1} \subset V_{1} \forall v_{t} \in V_{2}^{1}$ and $V_{a}^{1} \subset V_{1} \forall v_{1} \in V_{a}^{1} \times$ Since $V_{2}^{1} \cap V_{a}^{1}=\emptyset$, then for every vertex in $V_{a}^{1}$ and $V_{2}^{1}$ which are incident to exactly one vertex of $V_{1}$. Hence $V_{2}^{1} \cup V_{a}^{1}$ 
is a independent set in $G$ which gives $\left|V_{2}^{1} \cup V_{a}^{1}\right|=\beta_{0}(G)$. As in case 1 , which gives $|H|=\gamma(G)$ and $\left|H_{1}\right|=\gamma_{s b s}(G)$. Clearly $\left|H_{1}\right|+|H| \geq\left|V_{2}^{1}(G) \cup V_{a}^{1}(G)\right|-1$ which gives the above result.

Theorem 14: For any connected graph $G,[B S(G)] \neq K_{p}$, then $\gamma_{s b s}(G)+\Delta(G) \geq \beta_{1}(G)$ where $\beta_{1}(G)$ is the maximum edge independence number of $G$.

Proof: By the definition of split domination, we considered the graphs with the property that $[B S(G)] \neq K_{p}$. Let $E=\left\{e_{1}, e_{2}, e_{s} \ldots \ldots \ldots \theta_{n 1}\right\} \subseteq E(G)$ be the maximal set of edges with $N\left(e_{i}\right) \cap N\left(e_{j}\right)=e_{x}$ for every $e_{i}, e_{j} \in E, 1 \leq i, j \leq n$ and $e \in E(G)-E$. Clearly $E$ forms a maximal independent edge set in $G$. Then $|E|=\beta_{1}(G)$. Let $S=\left\{B_{1} B_{2}, B_{2}, \ldots \ldots \ldots B_{n}\right\}$ be the blocks in $G$ and $S_{1}=\left\{B_{1}{ }^{1} B_{2}{ }^{1}{ }_{0} B_{a}{ }^{1} \ldots \ldots \ldots B_{m}{ }^{1}\right\}$ be the blocks in $S(G)$. Let $V[B S(G)]=\left\{b_{1}{ }^{1}, b_{2}{ }^{1}, b_{a}{ }^{1}, \ldots \ldots \ldots b_{m}{ }^{1}\right\}$ be the set of vertices which corresponds to the blocks of $S_{1}$. Let $D$ be a dominating set of $[B S(G)], \forall v_{i} \in D_{x}$, then there exist $H=V[B S(G)]-D$ a disconnected graph. Hence $D$ is minimal split dominating set in [BS(G)]. Hence $|D|=\gamma_{s b s}(G)$. Now for a vertex $v \in V(G)$ with $\operatorname{deg}(v)=\Delta(G)$ then $|D|+\Delta(G) \geq|E|$ which gives $\gamma_{s b s}(G)+\Delta(G) \geq \beta_{1}(G)$.

\section{Nordhaus-Gaddum type results}

Theorem 15: For any graph $G$ with $n-$ blocks, $[B S(G)] \neq K_{p}$ and $[B S(\bar{G})] \neq K_{p}$, then i) $\gamma_{s b s}(G)+\gamma_{s b s}(\bar{G}) \leq 2 n$. iii) $\gamma_{s b s}(G) \times \gamma_{s b s}(\bar{G}) \leq n^{2}$.

\section{Conclusion}

In this paper we found an upper bound for block split subdivision domination in graphs in terms of blocks, domination, end blocks, cut vertices, minimum number of vertices, edge covering of $G$ and maximum independence number of $G$. Further the sharp lower bounds were found interms of cototal domination, total domination and maximum independence (vertex/edge) number of $G$. Finally Nordhaus-Gaddum type results are also found.

\section{References}

1. C. Berge, The Theory of Graphs (Mineola, New York, 2001).

2. O. Ore, Theory of Graphs, Am. Math. Soc. Colleq. Pub. 270 (1962).

3. G. Chartrand and P. Zhang, Introduction to Graph Theory (Tata McGraw-Hill Pub. Co., New Delhi, India, 2006)

4. F. Harary, Graph Theory (Adision-Wesley Publishing Co., London, 1972).

5. T. W. Haynes, S. T. Hedetniemi, and Petter J. Slatter, Fundamentals of Domination in Graphs, (Marcel Dekker, Inc, New York, 1988).

6. V. R. Kulli, Theory of Domination in Graphs (Vishwa International Publications, Gulbarga, India, 2010).

7. M. H. Muddebihal, S. Ahmed, and P. Shekanna, Int. J. Res. Eng. Technol. 2, 756 (2013).

8. M. H. Muddebihal and A. Majeed, Mathematical Theory Modeling 2, 10 (2012).

9. A. Majeed and M. H. Muddebihal, Int. J. Graph Theory 1, 9 (2013). 